Artigo / Article

\title{
Alterações moleculares no Mieloma Múltiplo
}

\section{Molecular abnormalities in Multiple Myeloma}

Esteban Braggio ${ }^{1}$

Ilana Z. Renault ${ }^{2}$

\begin{abstract}
Nos últimos anos tem acontecido uma revolução no conhecimento dos mecanismos moleculares envolvidos na patogênese do mieloma múltiplo, principalmente devido à incorporação de técnicas de citogenética molecular (hibridização in situ fluorescente FISH) e biologia molecular (Reação em cadeia da polimerase - PCR, hibridização genômica comparativa baseada em microarranjos - aCGH e microarranjos de RNA). Estas técnicas permitem estudar as células em interfase e detectar alterações cromossômicas crípticas, superando assim problemas metodológicos relacionados à análise por citogenética convencional. Por outro lado, a complexidade metodológica relacionada com a realização das técnicas de FISH e biologia molecular tem impossibilitado que sejam amplamente utilizadas na rotina laboratorial. Este trabalho tem como objetivo fazer uma revisão das principais vias moleculares alteradas no mieloma múltiplo, o seu valor prognóstico e a sua utilização na estratificação dos pacientes em diferentes grupos de risco. São detalhadas diferentes abordagens metodológicas e uma ênfase especial é dada em relação à importância da análise se restringir exclusivamente às células plasmocitárias. Por último, discutimos uma série de prioridades e estratégias de estudo na rotina laboratorial. Rev. bras. hematol. hemoter. 2007;29(1):10-16.
\end{abstract}

Palavras-chave: Mieloma múltiplo; alterações moleculares; FISH.

\section{Genética e citogenética do mieloma múltiplo}

As células neoplásicas do mieloma múltiplo (MM) apresentam uma complexa combinação de alterações genéticas e citogenéticas. ${ }^{1-3}$ Inicialmente achou-se que estas alterações surgiam de forma caótica e aleatória e não se conheciam as interações entre elas. No entanto, achados recentes têm contribuído ao reconhecimento de várias destas alterações como sendo marcadores biológicos relacionados com diferentes subgrupos de doentes e apresentarem valor prognóstico para a tomada de decisões clínicas. Além disso, estão sendo realizadas as primeiras tentativas de uma estratificação em grupos de risco baseada na caracterização molecular dos pacientes.

\section{Ploidia e translocações do gene da cadeia pesada das imunoglobulinas (IgH) \\ No MM a ocorrência de aneuploidia é característica, ${ }^{1-9}$}

sendo a sua presença independente do estádio da doença. ${ }^{3,4,10}$ Considerando a ploidia, quatro categorias podem ser distinguidas nos pacientes com MM: hipodiploidia (45 cromossomos), pseudodiploidia (46 cromossomos, mas com perdas e ganhos em relação ao cariótipo original), hiperdiploidia (47-74 cromossomos) e hipotetraploidia ou quase tetraplóide (75 ou mais cromossomos). ${ }^{8,11-13}$ As quatro categorias anteriormente mencionadas podem ser condensadas em dois grupos principais: hiperdiplóide e não-hiperdiplóide, no qual se incluem as outras três categorias. Esta classificação está baseada em que o quase tetraplóide parece representar genomas de $4 \mathrm{~N}$ presentes em células que possuem cariótipos pseudodiplóides ou hipodiplóides., ${ }^{8,11,13}$

O grupo composto pelos hiperdiplóides apresenta trissomias múltiplas, envolvendo principalmente os cromossomos 3, 5, 7, 9, 11, 15, 19 e 21. ${ }^{1-6,8,14}$ Por outro lado, foi identificado que o grupo não-hiperdiplóide possui um nú-

\footnotetext{
Correspondência: Esteban Braggio, Ilana Zalcberg Renault

Laboratório de Biologia Molecular, Cemo, INCa.

Praça da Cruz Vermelha 23, $6^{\circ}$ andar

20230-130 - Rio de Janeiro-RJ - Brasil

Tel (21) 2506-6506 - Fax (21) 2509-2121

E-mail:braggio.esteban@mayo.edu; zalcberg@inca.gov.br
}

${ }^{1}$ Mestre em Genética; PhD em Clínica Médica. Laboratório de Biologia Molecular - Cemo, INCa.

${ }^{2} \mathrm{PhD}$ em Hematologia Molecular. Chefe do Laboratório de Biologia Molecular. Servico de Atividades Laboratoriais - Cemo, INCa 
mero consideravelmente superior de translocações primárias envolvendo o gene da cadeia pesada das imunoglobulinas IgH quando comparado com os hiperdiplóides ( $>85 \%$ e $<30 \%$ dos pacientes, respectivamente). ${ }^{8,15,16}$

Uma observação similar foi obtida a partir do estudo da deleção do cromossomo 13 (72\% em pacientes não-hiperdiplóides versus $37 \%$ em hiperdiplóides). ${ }^{8,15}$ Além disso, o MM não-hiperdiplóide possui uma maior prevalência de alterações cromossômicas estruturais quando comparado ao hiperdiplóide (8,7 versus 4,7 quebras cromossômicas por cariótipo). ${ }^{15}$

Baseados nas observações anteriores podem-se distinguir duas vias patogênicas principais no desenvolvimento das células tumorais: a) os tumores não-hiperdiplóides, nos quais as translocações do $I g H$ representariam o evento inicial e universal relacionado com a imortalização do clone maligno; b) os tumores hiperdiplóides, nos quais outro mecanismo estaria relacionado com o evento inicial, ainda desconhecido, mas provavelmente inerente à aquisição da hiperdiploidia. ${ }^{8,13}$

\section{Translocações envolvendo os loci das imunoglobulinas}

As translocações de $\mathrm{IgH}$ parecem ser eventos iniciais e fundamentais na patogênese das neoplasias de células plasmocitárias. ${ }^{17,18}$ Foi demonstrado que as translocações de IgH encontram-se em aproximadamente $50 \%$ dos pacientes com gamopatias monoclonais de significado indeterminado (GMSI) e MM intramedular, aumentando a sua prevalência nos estádios mais avançados da doença, estando presentes em aproximadamente $80 \%$ das leucemias primárias de células plasmocitárias(LCP) e em até 90\% das linhagens celulares. ${ }^{16,19,20}$ Alterações envolvendo o lócus IgL apresentam freqüências dentre 10\% e 20\% em GMSI e MM intramedular, respectivamente. ${ }^{21}$

A presença de duas ou mais translocações de $\mathrm{IgH}$ e/ou IgL são eventos raros no MM, mas são detectadas em numero considerável nas linhagens celulares, as quais são originadas a partir de LCP ou de MM extramedulares..$^{22}$ Ainda não está clara a relação entre o acúmulo de translocações e a progressão da doença, no entanto o aumento na prevalência de alterações nos estádios extramedulares da doença sugere que as translocações poderiam fornecer um efeito proliferativo ou de sobrevida que facilitaria a progressão da doença a uma fase extramedular independente do estroma. ${ }^{19,20,23}$

\section{Diversidade de loci cromossômicos envolvidos nas translocações de imunoglobulinas}

Em contraste com o observado em outras neoplasias B, no MM existe uma diversidade considerável de loci cromossômicos envolvidos nas translocações com as imunoglobulinas. Estas translocações resultam na co-localização dos loci $\operatorname{IgH}$ ou $I g L$ e proto-oncogenes localizados nos cromossomos parceiros envolvidos. A maioria destes proto-oncogenes possui funções relacionadas com proliferação celular e fatores de transcrição, sendo superexpressos nos cromossomos derivados da translocação devido a sua localização próxima de regiões promotoras e enhancers dos loci Ig. ${ }^{24}$ Cinco regiões estão envolvidas em pelo menos 3\% dos tumores: 11q13 ( 15\% dos casos, sítio de localização da ciclina D1), 4p16 ( 15\%, FGFR3 e MMSET), 16q23 ( 6\%, c-maf), 6p21 ( 4\%, ciclina D3) e 8q24 ( 3\%, c-myc). Também foram identificados como loci recorrentes 20q11 ( maf-B) e 6p25 (IRF-4/MUM-1), mas em menor freqüência. Finalmente, em 20\%-30\% dos tumores o cromossomo parceiro apresenta uma prevalência inferior a $1 \%$.

\section{$t(11 ; 14)(q 13 ; q 32)$}

A t(11;14)(q13;q32) resulta na expressão ectópica da ciclina D1. ${ }^{25}$ Existe uma associação entre a presença da alteração e MM de cadeia leve ou não secretor, expressão de CD20 e morfologia linfoplasmocítica. ${ }^{26-28} \mathrm{~A}$ alteração também é detectada em alta freqüência em amiloidose (50\%) e mieloma $\operatorname{IgM}(90 \%) .{ }^{29,30}$ As conseqüências biológicas desta alteração permanecem desconhecidas, no entanto foi associada com uma menor capacidade proliferativa das células envolvidas.

Em relação ao seu valor prognóstico, a t(11;14) (q13;q32) estaria associada com um aumento na sobrevida, sobretudo em pacientes tratados com quimioterapia de altas doses. ${ }^{23,27,31,32}$ No entanto, o seu valor favorável ainda está em discussão, já que não foi demonstrado o aumento na prevalência da translocação em sobreviventes com longo follow-up.

\section{$\mathrm{t}(4 ; 14)(\mathrm{p} 16.3 ; \mathrm{q} 32)$}

A t(4;14)(p16.3;q32) é uma alteração citogenética críptica, que somente foi detectada após experimentos de clonagem em linhagens celulares de MM. . $^{33,34} \mathrm{O}$ estudo do ponto de quebra permitiu utilizar as técnicas de FISH e RTPCR na sua detecção de forma rotineira, sendo identificada em aproximadamente $15 \%$ dos casos com MM e $25 \%$ das linhagens celulares..$^{31,33-36} \mathrm{O}$ ponto de quebra na região $4 \mathrm{p} 16$ está localizado entre os proto-oncogenes FGFR3 e MMSET; assim, a translocação levaria à superexpressão dos dois oncogenes. Em aproximadamente 25\% dos casos parece ocorrer uma translocação desbalanceada com perda do der(14), ou permanência do der(14), mas com perda de expressão de FGFR3. ${ }^{37,38}$ Por outro lado, a perda do der(4) não foi observada. Estas observações sugerem que a persistência da expressão de MMSET seria um evento fundamental na perpetuação da expansão clonal, embora não se conheça ainda a sua função. Em etapas mais avançadas da doença foram identificadas mutações de ponto no gene FGFR3.

Existe uma associação entre os pacientes com a alteração e mieloma IgA ou de cadeia leve $\lambda$. $^{23,31}$

A presença da t(4;14)(p16.3;q32) é um fator prognóstico desfavorável independente em pacientes recebendo quimioterapia convencional ou de altas doses..$^{23,31,37}$ A presença 
da alteração também foi associada com uma sobrevida total $(p=0,005)$ e livre de eventos $(p=0,0001)$ menor em pacientes que receberam transplante autólogo de precursores hematopoiéticos. ${ }^{39}$

\section{$\mathbf{t}(14 ; 16)(\mathrm{q} 32 ; \mathrm{q} 23)$}

A t(14;16)(q32;q23) é detectada em 2\%-5\% dos pacientes com MM e em aproximadamente $25 \%$ das linhagens celulares. ${ }^{19,23,40}$ Esta alteração é difícil de se identificar por citogenética convencional e a incorporação do FISH aperfeiçoou a sua deteç̧ão.

Assim como a t( $4 ; 14)$, a presença da $\mathrm{t}(14 ; 16)$ foi associada com um prognóstico altamente desfavorável. ${ }^{23}$

\section{Implicância clínica das categorias de ploidia}

Duas associações têm sido identificadas considerando o nível de ploidia e o desfecho clínico. Por um lado, a presença de trissomias tem sido associada a um prognóstico mais favorável, provavelmente porque estão relacionadas à variante hiperdiplóide, a qual tem sido associada com uma maior sobrevida.$^{41}$ Por outro lado, a hipodiploidia está associada com um prognóstico desfavorável, ${ }^{8,13,42-44}$ o qual possivelmente se deve à presença significativamente superior de translocações no lócus $\operatorname{IgH} .^{15,16}$

Assim, a presença de hipodiploidia é um marcador prognóstico poderoso que possui implicações prognósticas independentes. ${ }^{8,13,43}$ No entanto, a contribuição específica e a significância na patogênese da hipodiploidia ainda não foram elucidadas.

\section{Anormalidades do cromossomo 13}

A deleção da região 13q14 (-13q14) apresenta uma alta prevalência no MM e em outras doenças de células plasmocitárias. ${ }^{45,46}$ Utilizando-se técnicas citogenéticas, a -13q14 foi detectada em 10\%-20\% dos pacientes com MM, ${ }^{7,47}$ sendo que, com a incorporação da técnica de FISH, essa porcentagem aumentou para $40 \%-50 \% .{ }^{48-50}$ Shaughnessy e cols. ${ }^{51}$ cobrindo o cromossomo 13 inteiro com 11 sondas diferentes, detectaram a deleção em $86 \%$ dos casos; no entanto, esses resultados não foram confirmados por outros estudos. A incorporação da técnica de FISH também permitiu detectar a alteração em estádios anteriores como GMSI e mieloma múltiplo assintomático, previamente não identificados devido à dificuldade na obtenção de metáfases clonais.

A -13q14 é um fator prognóstico desfavorável independente associado com uma menor sobrevida e resposta terapêutica, independentemente do tipo de tratamento (quimioterapia convencional ou de altas doses) ou método de deteç̧ão utilizado (citogenética convencional ou FISH). ${ }^{41,49,50,52-55}$ Contudo, é importante ressaltar que o efeito da -13q14 na sobrevida é mais desfavorável quando detectada por citogenética do que quando detectada por FISH. ${ }^{49,50,52,54-57}$ Este achado deve-se aos efeitos aditivos no prognóstico da detecção de metáfases anormais, o que indi- ca uma maior carga tumoral e um clone em fase proliferativa, relacionados com um estádio mais avançado da doença.

O estudo da -13q14 foi integrado na prática clínica nos últimos cinco anos, no entanto muitas questões ainda não foram resolvidas. Assim, a comparação retrospectiva dos dados de dois estudos indica que o benefício da quimioterapia de altas doses é maior em pacientes sem a -13q14 detectada por FISH, sugerindo o beneficio limitado deste tratamento em pacientes com a deleção. ${ }^{50,52}$ Adicionalmente foi observado que o uso de interferon- $\alpha$ em pacientes com -13q14 resultou em uma sobrevida menor. ${ }^{50}$

\section{Outras alterações genéticas com valor prognóstico}

\section{Mutações nos genes Ras}

Mutações de ponto têm sido identificadas nos códons 12, 13 e 61 de K-ras ou N-ras. As mutações são eventos muito raros nas GMSI, mas estão presentes em 35\%-50\% dos pacientes com MM, podendo aumentar a prevalência nos estágios mais avançados da doença. ${ }^{58,59}$ Estes dados sugerem que alterações em Ras podem ser um marcador molecular da progressão de GMSI para MM. ${ }^{58,60}$ A presença de mutações em Kras ou N-ras esteve associada com uma menor sobrevida. ${ }^{57}$

Em linhagens com a t $(4 ; 14)$ foram observadas mutações ativadoras em Ras e FGFR3 como sendo eventos mutuamente excludentes, o que sugere que as alterações nessas duas vias produziriam um efeito similar na patogênese. ${ }^{61}$

\section{Inativação de p53}

A inativação de $p 53$ por mutação ou por deleção da região 17p13 parece ser um evento raro nas GMSI e no MM ao diagnóstico, sendo restrito aos últimos estádios da doença. ${ }^{62-64}$ As alterações estão presentes em $5 \%$ dos pacientes ao diagnóstico, 20\%-40\% em MM avançado ou LCP e em $60 \%$ de linhagens celulares. ${ }^{65,66}$

A -17q13 é um fator prognóstico desfavorável independente de sobrevida livre de eventos e sobrevida total em pacientes tratados com quimioterapia convencional, de altas doses ou transplante autólogo de células precursoras hematopoiéticas. ${ }^{23,61,67}$ Além disso, a deleção está associada com outras características clínicas de prognóstico desfavorável como hipercalcemia, altos níveis de creatinina sérica e plasmocitomas extramedulares. ${ }^{1,9}$

\section{Anormalidades no cromossomo 1}

Alterações no cromossomo 1 são eventos muito comuns em neoplasias hematológicas e constituem a principal alteração estrutural no MM, sendo observadas em 48\% dos pacientes. ${ }^{7,68}$ Foi sugerido que as alterações estruturais estariam associadas com uma taxa de divisão celular elevada e com uma menor sobrevida. ${ }^{8}$ Por outro lado, a amplificação ou a deleção do braço 1q estariam associadas com um fenótipo mais agressivo da doença. ${ }^{69}$ 


\section{Classificação molecular}

Várias classificações têm sido propostas baseadas na presença de alterações genéticas e citogenéticas. As duas principais encontram-se abaixo relacionadas.

\section{Classificação baseada nas alterações citogenéticas com valor prognóstico diferencial}

Um estudo realizado em 351 pacientes tratados com quimioterapia convencional permitiu identificar três categorias de risco baseando-se na presença de alterações cromossômicas identificadas pela técnica de FISH. ${ }^{23}$

a) Grupo de pior prognóstico: a presença da $\mathrm{t}(4 ; 14)$ (p16;q32), t(14;16)(q32;q23) ou -17p13 foi associada com uma sobrevida menor ( $p<0,001, p=0,003$ e $p=0,005$, respectivamente). A mediana de sobrevida dos pacientes possuindo pelo menos uma destas alterações foi de 25 meses.

b) Grupo de prognóstico intermediário: este grupo esteve composto por pacientes que apresentaram a -13q14. A presença desta alteração também esteve associada com uma menor sobrevida ( $p=0,028)$, mas apresentou uma mediana de sobrevida consideravelmente maior (42 meses).

c) Grupo de bom prognóstico: este grupo incluiu os pacientes restantes, incluso aqueles que apresentaram a $\mathrm{t}(11 ; 14)(q 13 ; \mathrm{q} 32)$. A mediana de sobrevida foi de 51 meses.

\section{Classificação TC (translocações de IgH e ciclinas)}

A partir de estudos do perfil de expressão gênica tem sido sugerido que o nível de expressão de pelo menos um gene da família das ciclinas D seria superior aos observados em plasmócitos normais em virtualmente todos os pacientes com MM, incluindo aqueles sem translocações envolvendo o $\operatorname{IgH}^{24,70,71}$

Assim, como uma conseqüência direta das translocações de imunoglobulinas, a ciclina D1 seria superexpressa em associação com a t(11;14)(q13;q32) e a D3 associada com a t(6;14)(p21;q32). Por outro lado, a D2 seria superexpressa como conseqüência indireta das $t(4 ; 14)(p 16 ; q 32)$ e $\mathrm{t}(14 ; 16)(\mathrm{q} 32 ; 23)$.

Dos pacientes que não possuem translocações, mais da metade apresenta expressão ectópica da D1. O mecanismo de expressão é desconhecido, mas foi sugerido que alterações numéricas no cromossomo 11 seriam as responsáveis da elevação dos níveis de RNAm. ${ }^{72}$ A maioria dos pacientes restantes expressaria altos níveis de D2 e somente uma pequena proporção apresentaria níveis de expressão normais das três ciclinas. Assim, a desregulação dos genes das ciclinas D seria um evento unificador e precoce dentro da neoplasia, deixando as células mais susceptíveis ao estímulo proliferativo, resultando na expansão seletiva do clone maligno.

A partir dos achados anteriores, foi proposta a classificação molecular TC dos MM baseada na presença das translocações $I g H$ e a expressão das ciclinas D (tabela 1). ${ }^{73}$

\section{Implicações da classificação TC}

Embora esta classificação ainda esteja em fase de teste, já foram identificados achados clínicos e biológicos que se correlacionam com diferentes grupos de pacientes. Assim, o grupo D1 está ausente nas LCP e linhagens celulares, sugerindo que estes tumores apresentam uma forte dependência do estroma intramedular. Por outro lado, as lesões ósseas líticas estão presentes em uma alta prevalência ( 90\%) nos grupos 6p21, 11q13, D1 e D1+D2, mas apresentam valores significativamente inferiores ( $\sim 55 \%)$ nos grupos 4 p16 e maf. $^{71}$

Pacientes do grupo $\mathrm{t}(4 ; 14)$ e do $\mathrm{t}(14 ; 16)$ apresentam uma menor sobrevida quando tratados com quimioterapia convencional ou de altas doses, enquanto pacientes do grupo t $(11 ; 14)$ apresentam uma melhor sobrevida. Similarmente, os pacientes do grupo D1, principalmente associados com hiperdiploidia, apresentam melhor prognóstico. Do grupo D1+D2 não se possuem muitos dados, no entanto está associado com um índice proliferativo elevado e com pacientes em recaída, pelo que os primeiros indícios indicam que seria de prognóstico desfavorável. O grupo sem alterações nem superexpressão de ciclinas é muito pequeno para se obterem conclusões, mas, devido ao fato de estar associado a pacientes com doença macrofocal, poderia ser classificados inicialmente como de bom prognóstico.

Os resultados anteriores sugerem que a classificação TC poderia ser utilizada na clínica para separar pacientes em grupos com distintos subtipos de MM.

\section{Abordagens metodológicas}

\section{Citogenética}

O estudo do MM por citogenética se vê dificultado principalmente pelo baixo índice mitótico da doença, conseguindo-se metáfases somente em 20\%-30\% dos casos. Além disso, a técnica não permite diferenciar as células tumorais das restantes populações celulares. Isto leva muitas vezes a resultados falsos negativos baseados na análise cariotípica de células
Tabela 1. Subgrupos moleculares de pacientes baseados na classificação TC (translocações de $\operatorname{lgH}$ e ciclinas D).73

\begin{tabular}{cccccc}
\hline Grupo TC & $\begin{array}{c}\text { Translocação } \\
\text { primária }\end{array}$ & $\begin{array}{c}\text { Ciclina D } \\
\text { desregulada }\end{array}$ & Ploidia & $\begin{array}{c}\text { Freqüência } \\
(\%)\end{array}$ & Prognóstico \\
\hline 6p21 & $6 p 21$ & $\mathrm{D} 3$ & $\mathrm{NH}$ & 3 & Bom? \\
11q13 & $11 q 13$ & $\mathrm{D} 1$ & $\mathrm{D}=\mathrm{NH}$ & 16 & Bom \\
D1 & Ausente & $\mathrm{D} 1$ & $\mathrm{H}$ & 34 & Bom \\
D1+D2 & Ausente & $\mathrm{D} 1+\mathrm{D} 2$ & $\mathrm{H}$ & 6 & Ruim? \\
D2 & Ausente & $\mathrm{D} 2$ & $\mathrm{H}=\mathrm{NH}$ & 17 & $?$ \\
Nenhum & Ausente & Nenhuma & $\mathrm{NH}$ & 2 & Bom? \\
4p16 & $4 p 16$ & $\mathrm{D} 2$ & $\mathrm{NH}>\mathrm{H}$ & 15 & Ruim \\
Maf & 16q23/20q11 & $\mathrm{D} 2$ & $\mathrm{NH}$ & 7 & Ruim \\
\hline
\end{tabular}

NH: não-hiperdiplóide; D: diplóide; H: hiperdiplóide. 
mielóides em divisão presentes na medula óssea. Por outro lado, esta técnica não detecta alterações crípticas como a $\mathrm{t}(4 ; 14)(\mathrm{p} 16 ; \mathrm{q} 32)$, e raramente detecta a t(14;16) (q32;q23) e a -17p13. Por todas as considerações anteriores, a sua utilização foi amplamente substituída pela técnica de FISH. Contudo, continua sendo uma abordagem recomendada para a identificação da -13q14, a qual, quando detectada por citogenética, está relacionada com um pior prognóstico que quando detectada por FISH. Também é uma ferramenta adequada para a estudo de alterações cromossômicas numéricas.

\section{FISH}

A técnica de FISH é atualmente a metodologia mais utilizada no estudo das alterações cromossômicas no MM. Esta pode ser realizada a partir de células em interfase, eliminando as limitações metodológicas da citogenética relacionadas com o baixo índice proliferativo. A partir da sua introdução mudou a caracterização molecular da doença, aumentando a freqüência de deteç̧ão das alterações consideravelmente. Assim, por exemplo, a freqüência da -13q14 aumentou de 10\%-15\% para 40\%-50\%. Além disso, permitiu a identificação da $\mathrm{t}(4 ; 14)(\mathrm{p} 16 ; \mathrm{q} 32)$, previamente desconhecida.

É de fundamental importância que a análise seja realizada exclusivamente a partir de células plasmocitárias, para o qual duas estratégias podem ser utilizadas:

a) Purificação celular: utilizando-se anticorpos antiCD138 pode ser realizada a separação positiva dos plasmócitos por meio de colunas magnéticas ou sorting. A grande limitação desta abordagem é a perda considerável de células durante o processo de purificação.

b) cIg-FISH: a combinação da técnica de FISH com a detecção das células plasmáticas por meio de anticorpos fluorescentes anticadeia leve ou citoplasmáticas permite identificar os plasmócitos e realizar a análise exclusivamente nessa população. Somente são necessárias 100 células plasmocitárias para se avaliar o caso.

\section{RT-PCR}

A identificação do ponto de quebra do cromossomo 4 na t(4;14)(p16;q32) permitiu utilizar a técnica de RT-PCR para a sua detecção de forma rotineira. Esta técnica apresenta a alta sensibilidade própria da reação de PCR, eliminando a necessidade da separação da população tumoral. Além disso, nos casos positivos, a detecção da translocação pode ser utilizada como marcador no acompanhamento da doença residual mínima.

\section{Recomendações}

Contamos com informações suficientes para realizar recomendações referentes aos testes moleculares a serem incluídos na rotina em pacientes com MM. Recentemente foi proposta a inclusão na rotina da deteç̧ão das $\mathrm{t}(4 ; 14)$ (p16;q32), t(11;14)(q13,q32), t(14;16)(q32,q23) e -17p13 pela técnica de
FISH e da -13q14 e do nível de ploidia pela citogenética convencional. ${ }^{74}$ Todas estas alterações foram associadas a um prognóstico desfavorável à exceção da t(11;14).

Embora o conhecimento destas alterações seja muito importante na estratificação dos pacientes em diferentes grupos de risco, nós propomos determinadas prioridades e abordagens alternativas:

- $\quad t(4 ; 14):$ a deteç̧ão desta alteração é prioritária por estar associada com pacientes pertencentes ao grupo de alto risco e não respondedores às terapias atualmente em uso. Este grupo de pacientes constitui um alvo de novas terapias potenciais baseadas em inibidores de FGFR3. A caracterização do sítio de quebra permite a sua detecção por meio do RT-PCR, sendo uma técnica mais sensível e acessível que o FISH.

- -13q14: embora a detecção por citogenética convencional esteja associada com um pior prognóstico que quando detectada por FISH, a baixa proporção de casos com metáfases dificulta a realização da técnica. Por outro lado, a presença da alteração por FISH continua sendo um fator prognóstico desfavorável. Sugerimos a análise simultânea por ambas as técnicas.

- Ploidia: o valor prognóstico desfavorável dos mielomas não-hiperdiplóides parece ser uma conseqüência indireta da alta proporção de casos com translocações de risco. As alterações cromossômicas numéricas podem ser estudadas por citogenética convencional.

- $t(11 ; 14)$ : a técnica ideal para o seu estudo é o FISH. No entanto, o seu valor prognóstico favorável coloca o seu estudo em um nível inferior de prioridades que as alterações anteriores.

- $t(14 ; 16)$ e -17p13: a presença de ambas está associada com o grupo de alto risco. No entanto, a sua freqüência extremamente baixa (2-5\%) não torna a sua inclusão prioritária na rotina laboratorial.

- Amplificação do 1q: estudos recentes têm sugerido que a amplificação do braço 1q estaria associada com um valor prognóstico altamente desfavorável. Estes dados devem ser confirmados por estudos adicionais antes de incluir esta alteração na rotina laboratorial.

\section{Abstract}

In recent years, we have seen an explosion in knowledge on genetic and cytogenetic mechanisms involved in the pathogenesis of multiple myeloma, mainly due to the incorporation of molecular tools in its study (fluorescence in situ hybridization - FISH, Real time PCR and RNA microarrays). These tools have enabled the study of cells in interphase and to detect cryptic chromosomal abnormalities, replacing cytogenetic techniques. The technical complexities associated with the performance of these tests, however, have limited their widespread applicability in routine clinical practice. The aim of this work is to review the present status of our knowledge of the pathogenetic pathways of the disease, the prognostic value of the main chromosomal abnormalities and its role in risk stratification of multiple myeloma patients. We detail technical aspects, emphasizing the importance to 
restrict analysis to myeloma cells. Finally, we discuss different strategies and recommendations for the adoption of routine molecular genetic testing. Rev. bras. hematol. hemoter. 2007;29(1):10-16.

Key words: Multiple myeloma; molecular abnormalities; FISH.

\section{Referências Bibliográficas}

1. Dewald G, Kyle R, Hicks G, et al. The clinical significance of cytogenetic studies in 100 patients with multiple myeloma, plasma cell leukemia, or amyloidosis. Blood 1985;66:380-90.

2. Tabernero D, Miguel JS, Garcia-Sanz M, et al. Incidence of chromosome numerical changes in multiple myeloma: fluorescence in situ hybridization analysis using 15 chromosome-specific probes. Am J Pathol 1996;149:153-61.

3. Zandecki M, Lai J, Facon T. Multiple myeloma: almost all patients are cytogenetically abnormal. Br J Haematol 1996;94:217-27.

4. Drach J, Schuster J, Nowotny H, et al. Multiple myeloma: high incidence of chromosomal aneuploidy as detected by interphase fluorescence in situ hybridization. Cancer Research 1995; 55: 3.854-9.

5. Fonseca R, Ahmann G, Juneau A, et al. Cytogenetic abnormalities in multiple myeloma and related plasma cell disorders; a comparison of conventional cytogenetic analysis to fluorescent in situ hybridization with simultaneous cytoplasmic immunoglobulin staining. Blood 1997;90:1.349.

6. Lai J, Zandecki M, Mary J, et al. Improved cytogenetics in multiple myeloma: a study of 151 patients including 117 patients at diagnosis. Blood 1995;85:2.490-7.

7. Sawyer J, Waldron J, Jagannath S, et al. Cytogenetic findings in 200 patients with multiple myeloma. Cancer Genet Cytogenet 1995;82:41-9.

8. Debes-Marun C, Dewald G, Bryant S, et al. Chromosome abnormalities clustering and its implications for pathogenesis and prognosis in myeloma. Leukemia 2003;17:427-36.

9. Gould J, Alexanian R, Goodacre A, et al. Plasma cell karyotype in multiple myeloma. Blood 1988;71:453-6.

10. Zandecki M, Bernardi F, Lai J, et al. Image analysis in multiple myeloma at diagnosis. Correlation with cytogenetic study. Cancer Genet Cytogenet 1994;74:115-9.

11. Smadja N, Fruchart C, Isnard F, et al. Chromosomal analysis in multiple myeloma: cytogenetic evidence of two different diseases. Leukemia 1998;12:960-9.

12. Smadja N, Bastard C, Brigaudeau C. Primary plasma cell leukemia and multiple myeloma: one or two diseases according to the methodology. Blood 1999;94:3.607-9.

13. Smadja N, Bastard C, Brigaudeau C, et al. Hypodiploidy is a major prognostic factor in multiple myeloma. Blood 2001; 98:2.229-38.

14. Latreille J, Barlogie B, Johnston D, et al. Ploidy and proliferative characteristics in monoclonal gammopathies. Blood 1982; 59:43-51.

15. Smadja N, Leroux D, Soulier J, et al.Further cytogenetic characterization of multiple myeloma confirms that $14 \mathrm{q} 32$ translocations are a very rare event in hyperdiploid cases. Genes, Chromosomes Cancer 2003;38:234-9.

16. Fonseca R, Debes-Marun C, Picken E, et al. The recurrent IgH translocations are highly associated with nonhyperdiploid variant multiple myeloma. Blood 2003;102:2.562-7.

17. Bergsagel P, Chesi M, Brents L, et al. Translocations into IgH switch regions. The genetic hallmark of multiple myeloma. Blood 1995;86:223-33.

18. Bergsagel P, Chesi M, Nardini E, et al. Promiscuous translocations into immunoglobulin heavy chain switch regions in multiple myeloma. Proc Natl Acad Sci USA 1996;93:13.931-6.

19. Avet-Loiseau H, Facon T, Grosbois B, et al. Oncogenesis of multiple myeloma: 14q32 and 13q chromosomal abnormalities are not randomly distributed, but correlate with natural history, immunological features, and clinical presentation. Blood 2002; 99:2.185-91.

20. Nishida K, Tamura A, Nakazawa N, et al. The Ig heavy chain gene is frequently involved in chromosomal translocations in multiple myeloma and plasma cell leukemia as detected by in situ hybridization. Blood 1997;90:526-34.

21. Fonseca R, Bailey R, Ahmann G, et al. Genomic abnormalities in monoclonal gammopathy of undetermined significance. Blood 2002;100:1.417-24.

22. Bergsagel P, Kuehl W. Chromosome translocations in multiple myeloma. Oncogene 2001;20:5.611-22.

23. Fonseca R, Blood E, Rue M, et al. Clinical and biologic implications of recurrent genomic aberrations in myeloma. Blood 2003; 101:4.569-75.

24. Bergsagel P, Kuehl M. Critical roles for immunoglobulin translocations and cyclin D dysregulation in multiple myeloma. Immunol Rev 2003;194:96-104.

25. Chesi M, Bergsagel P, Brents L, et al. Dysregulation of cyclin D1 by translocation into an IgH switch region in two multiple myeloma cell lines. Blood 1996;88:674-81.

26. Robillard N, Avet-Loiseau H, Garand R, et al. CD20 is associated with a small mature plasma cell morphology and $t(11 ; 14)$ in multiple myeloma. Blood 2003;102:1.070-1.

27. Fonseca R, Blood EA, Oken MM, et al. Myeloma and the t(11;14) (q13;q32); evidence for a biologically defined unique subset of patients. Blood 2002;99:3.735-41.

28. Avet-Loiseau H, Garand R, Lode L, et al. Translocation t(11;14) (q13;q32) is the hallmark of IgM, IgE, and nonsecretory multiple myeloma variants. Blood 2003;101:1.570-71.

29. Harisson C, Mazullo H, Cheung K, et al. Chromosomal abnormalities in systematic amyloidosis. Proceedings of the VIII International Myeloma Workshop 2001:P18.

30. Hayman S, Bailey R, Jalal S, et al. Translocations involving heavychain locus are possible early genetic events in patients with primary systemic amyloidosis. Blood 2001;98:2.266-8.

31. Moreau P, Facon T, Leleu X, et al. Recurrent 14q32 translocations determine the prognosis of multiple myeloma, especially in patients receiving intensive chemotherapy. Blood 2002; 100:1.579-83.

32. Soverini S, Cavo M, Cellini C, et al. Cyclin D1 overexpression is a favorable prognostic variable for newly diagnosed multiple myeloma patients treated with high-dose chemotherapy and single or double autologous transplantation. Blood 2003; 102:1.588-94.

33. Chesi M, Nardini E, Lim R, et al. The $\mathrm{t}(4 ; 14)$ translocation in myeloma dysregulates both FGFR3 and a novel gene, MMSET, resulting in IgH/ MMSET hybrid transcripts. Blood 1998; 92: 3.025-34

34. Chesi M, Nardini E, Brents L, et al. Frequent translocation $\mathrm{t}(4 ; 14)(\mathrm{p} 16.3 ; \mathrm{q} 32.3)$ in multiple myeloma is associated with increased expression and activating mutations of fibroblast growth factor receptor 3. Nature Genetics 1997;16:260-4.

35. Fonseca R, Oken M, Greipp P. The t(4;14)(p16.3;q32) is strongly associated with chromosome 13 abnormalities in both multiple myeloma and monoclonal gammopathies of undetermined significance. Blood 2001;98:1.271-2.

36. Richelda R, Ronchetti D, Baldini L, et al. A novel chromosomal translocation $\mathrm{t}(4 ; 14)(\mathrm{p} 16.3 ; \mathrm{q} 32)$ in multiple myeloma involves the fibroblast growth-factor receptor 3 gene. Blood 1997; 90:4.062-70.

37. Keats J, Reiman T, Maxwell C, et al. In multiple myeloma, $\mathrm{t}(4 ; 14)(\mathrm{p} 16 ; \mathrm{q} 32)$ is an adverse prognostic factor irrespective of FGFR3 expression. Blood 2003;101:1.520-9.

38. Santra M, Zhan F, Tian E, et al. A subset of multiple myeloma harboring the $t(4 ; 14)(p 16 ; q 32)$ translocation lacks FGFR3 expression but maintains an IGH/MMSET fusion transcript. Blood 2003;101:2.374-6. 
39. Chang H, Sloan S, Li D, et al. The $\mathrm{t}(4 ; 14)$ is associated with poor prognosis in myeloma patients undergoing autologous stem cell transplant. Br J Haematol 2004;125:64-8.

40. Chesi M, Bergsagel P, Shonukan O, et al. Frequent dysregulation of the c-maf proto-oncogene at $16 q 23$ by translocation to an Ig locus in multiple myeloma. Blood 1998;91:4.457-63.

41. Perez-Simon J, Garcia-Sanz R, Tabernero M, et al. Prognostic value of numerical chromosome aberrations in multiple myeloma: a FISH analysis of 15 different chromosomes. Blood 1998; 91: 3.366-71.

42. Greipp P, Trendle M, Leong T, et al. Is flow cytometric DNA content hypodiploidy prognostic in multiple myeloma? Leuk Lymphoma 1999;35:83-9

43. Fassas A, Spencer T, Sawyer J, et al. Both hypodiploidy and deletion of chromosome 13 independently confer poor prognosis in multiple myeloma. Br J Haematol 2002;118:1.041-7.

44. Calasanz M, Cigudosa J, Odero M, et al. Hypodiploidy and 22q11 rearrangements at diagnosis are associated with poor prognosis in patients with multiple myeloma. Br J Haematol 1997;98:418-25.

45. Avet-Loiseau H, Li J, Morineau N, et al. Monosomy 13 is associated with the transition of monoclonal gammopathy of undetermined significance to multiple myeloma. Blood 1999;94:2.583-9.

46. Drach J, Kaufmann H, Urbauer E, et al. The biology of multiple myeloma. J. Cancer Res. Clin. Oncol 2000;126:441-7.

47. Dao D, Sawyer J, Epstein J, et al. Deletion of the Retinoblastoma gene in multiple myeloma. Leukemia 1994;8:1.280-4.

48. Avet-Loiseau H, Facon T, Daviet A, et al. 14q32 translocations and monosomy 13 observed in monoclonal gammopathy of undetermined significance delineate a multistep process for the oncogenesis of multiple myeloma. Cancer Research 1999; 59:4.546-50.

49. Zojer N, Konigsberg R, Ackermann J, et al. Deletion of 13q14 remains an independent adverse prognostic variable in multiple myeloma despite its frequent detection by interphase fluorescence in situ hybridization. Blood 2000;95:1.925-30.

50. Fonseca R, Harrington D, Oken M, et al. Biologic and prognostic significance of interphase FISH detection of chromosome 13 abnormalities in multiple myeloma: an Eastern Cooperative Oncology Group (ECOG) Study. Cancer Research 2002;62:715-20.

51. Shaughnessy J, Tian E, Sawyer J, et al. High incidence of chromosome 13 deletion in multiple myeloma detected by multiprobe interphase FISH. Blood 2000;96:1.505-11.

52. Facon T, Avet-Loiseau H, Guillerm G, et al. Chromosome 13 abnormalities identified by FISH analysis and serum beta-2microglobulin produce a powerful myeloma staging system for patients receiving high-dose therapy. Blood 2001;97:1.566-71.

53. Desikan R, Barlogie B, Sawyer J, et al. Results of high-dose therapy for 1000 patients with multiple myeloma: durable complete remissions and superior survival in the absence of chromosome 13 abnormalities. Blood 2000;95:4.008-10.

54. Shaughnessy J, Tian E, Sawyer J, et al. Prognostic impact of cytogenetic and interphase fluorescence in situ hybridizationdefined chromosome 13 deletion in multiple myeloma: early results of total therapy II. Br J Haematol 2003;120:44-52.

55. Seong C, Delasalle K, Hayes K, et al. Prognostic value of cytogenetics in multiple myeloma. Br J Haematol 1998;101:189-94.

56. Dewald G, Therneau T, Larson D, et al. Relationship of patient survival and chromosome anomalies detected in metaphase and/or interphase cells at diagnosis of myeloma. Blood 2005; 106: 3.553-8.

57. Worel N, Greinix H, Ackermann J, et al. Deletion of chromosome $13 q 14$ detected by fluorescence in situ hybridization has prognostic impact on survival after high-dose therapy in patients with multiple myeloma. Ann Hematol 2001;80:345-8.

58. Liu P, Leong T, Quam L, et al. Activating mutations of N- and Kras in multiple myeloma show different clinical associations: analysis of the Eastern Cooperative Oncology Group Phase III Trial. Blood 1996;88:2.699-706.

59. Bezieau S, Avet-Loiseau H, Moisan J, et al. Activating Ras mutations in patients with plasma-cell disorders: a reappraisal. Blood 2003; 100:1.101-2.

60. Bezieau S, Devilder M, Avet-Loiseau H, et al. High incidence of Nand K-Ras activating mutations in multiple myeloma and primary plasma cell leukemia at diagnosis. Hum. Mutat 2001; 18:212-24.

61. Chesi M, Brents L, Ely S, et al. Activated fibroblast growth factor receptor 3 is an oncogene that contributes to tumor progression in multiple myeloma. Blood 2001;97:729-36.

62. Drach J, Ackermann J, Fritz E, et al. Presence of a p53 gene deletion in patients with multiple myeloma predicts for short survival after conventional-dose chemotherapy. Blood 1998; 92:802-9.

63. Schultheis B, Kramer A, Willer A, et al. Analysis of p73 and p53 gene deletions in multiple myeloma. Leukemia 1999;13:2.099-103.

64. Neri A, Baldini L, Trecca D, et al. p53 gene mutations in multiple myeloma are associated with advanced forms of malignancy. Blood 1993;81:128-35.

65. Mazars G, Portier M, Zhang X, et al. Mutations of the p53 gene in human myeloma cell lines. Oncogene 1992;7:1.015-8.

66. Corradini P, Inghirami G, Astolfi M, et al. Inactivation of tumor suppressor genes, p53 and Rb1, in plasma cell dyscrasias. Leukemia 1994;8:758-67.

67. Gertz M, Lacy M, Dispenzieri A, et al. Clinical implications of $\mathrm{t}(11 ; 14)(\mathrm{q} 13 ; \mathrm{q} 32), \mathrm{t}(4 ; 14)(\mathrm{p} 16 ; \mathrm{q} 32)$, and $-17 \mathrm{p} 13$ in myeloma patients treated with high-dose therapy. Blood 2005;106:2.837-40.

68. Sawyer J, Tricot G, Mattox S, et al. Jumping translocations of chromosome 1q in multiple myeloma: evidence for a mechanism involving decondensation of pericentromeric heterochromatin. Blood 1998;91:1.732-41.

69. Inoue J, Otsuki T, Hirasawa A, et al. Overexpression of PDZK1 within the 1q12-q22 amplicon is likely to be associated with drug-resistance phenotype in multiple myeloma. Am J Pathol 2004; 165:71-81.

70. Zhan F, Hardin J, Kordsmeier B, et al. Global gene expression profiling of multiple myeloma, monoclonal gammopathy of undetermined significance and normal bone marrow plasma cells. Blood 2002;99:1.745-57.

71. Bergsagel PL, Kuehl M, Zhan F, et al. Cyclin D dysregulation: an early and unifying pathogenic event in multiple myeloma. Blood 2005;106:296-303.

72. Specht K, Haralambieva E, Bink K, et al. Different mechanisms of cyclin D1 overexpression in multiple myeloma revealed by fluorescence in situ hybridization and quantitative analysis of mRNA levels. Blood 2004;104:1.120-6.

73. Bergsagel PL, Kuehl M. Molecular Pathogenesis and a Consequent Classification of Multiple Myeloma. Journal of Clinical Oncology 2005;23:6.333-8.

74. Stewart K, Fonseca R. Prognostic and Therapeutic Significance of Myeloma Genetics and Gene Expression Profiling. Journal of Clinical Oncology 2005;23:6.339-44.

O tema apresentado e o convite ao(s) autor(es) consta da pauta elaborada pelo co-editor.

Avaliação: Co-editor e um revisor externo.

Publicado após revisão e concordância do editor.

Conflito de interesse: não declarado.

Recebido: 25/11/2006

Aceito: 05/01/2007 